C.A. Heusch

PPE Division, CERN

CH-1211 Geneva 23

$$
\text { and }
$$

University of California Sanra Cruz - CA 95064, U.S.A.

$$
\text { and }
$$

\section{P. Minkowski}

Institute for Theoretical Physic

University of Bern

CH - 3012 Bern, Switzerland

January 26,1995

\section{Abstract}

We discuss collisions of two equally charged leptons $\epsilon^{-} \epsilon^{-}$or $\epsilon^{+} \epsilon^{+}$, with $\ell=e, \mu$, where the exchange of heavy neutrino flavors induces the lepton-flavour-violating transitions to a pair of equally charged gauge bosons, $W^{-} W^{-}$or $W^{+} W^{+}$. In the center-of-mass energy tegion well above $W$ pair production threshold, but below the heavy neutrino mass, acceptable mass and mixing parameters for the heavy neutrino flavor lead to cross sections of a size and energy dependence that permit a clear detection of lepton flavor violation; the resulting signal constitutes a unique discovery channel for $\mathrm{TeV}$ level Majorana neutrinos.
The continued absence of an understanding why the three known neutrino flavors correspond to very small mass eigenstates, together with the need to embed our Standard Model low-energy phenomenology into a higher symmetry scheme, motivate us to look for evidence that might serve both purposes. In this letter, we will argue that it is natural to postulate the existence of at least two "heavy" neutrino flavors ( of mass $\gtrsim 1 \mathrm{TeV}$ ) and that there is an experimental scheme which is most likely the cleanest - if not the only - one that will permit us to prove their existence with experimental means presently in the planning stage.

Let us set the framework of our discussion by defining our lepton flavor scheme in terms of the left-handed fields

$$
\left(\begin{array}{ccccccc}
\nu_{1} & \nu_{2} & \nu_{3} & \mathcal{N}_{1} & \mathcal{N}_{2} & \ldots \\
e^{-} & \mu^{-} & \tau^{-} & e^{+} & \mu^{+} & \tau^{+} &
\end{array}\right) .
$$

Such a multiplet arises naturally within a broad class of models, such as, e.g., a primary unifying gauge group out of the chain E6 $\rightarrow$ SO10 $\rightarrow \cdots$, with three families of 27 representations of $E 6$.

We assume the breakdown of gauge invariance to generate the primary heavy neutrino flavor, of which we concentrate on the two least massive ones $\mathcal{N}_{1} \mathcal{N}_{2}$ with masses $M_{1,2}$, in addition to the three light ones. There will be nontrivial mixing between light and heavy flavors.

This scenario leads to a general bound for the largest of the invariant mixing angles between light and heavy flavors [1]. It is

$$
\left|\alpha_{\max }\right| \leq \frac{m_{i}^{(n)}}{\sqrt{M_{1} M_{2}}} .
$$

For exact or approximate Dirac degeneracy, $M_{1}, M_{2}$ are exactly or approximately equal. They are independent of the mixing angle in eq. (2), but are determined by the extended neutrino sector denoted by the dots in eq. (1), incorporating the appropriate version of the so called sea-saw mechanism.

$m_{i}^{(\mu)}$ denotes a mixing mass, the equivalent of the top quark mass, which bas been appropriately reduced, on the level of Yukawa couplings, to the neutrino sector. The crucial property of this mass scale is that it is independent of the heavy neutrino masses $M_{1}, \ldots$.

We estimate $m_{i}^{(\nu)} \sim 60 \mathrm{GeV}$ from the SO10 mass relation $m_{\nu j}=m_{u j}=$ $m_{e j}=m_{d j}$ (where $j=1,2,3$ is the family index ) which we assume to 
be valid at the unification scale, reflecting the dominance of the $(27)^{3}$ Yukawa couplings in generating all charged fermion masses of standard model fermions.

Existing limits on lepton flavor violating reactions and on the masses of light neutrino flavors just yield an order-of-magnitude estimate for the lower bound of the geometric mean $\sqrt{M_{1} M_{2}}[1],[2]$

$$
\sqrt{M_{1} M_{2}} \geq O(1-10 \mathrm{TeV})
$$

We are looking for a strategy to discover characteristic signatures due specifically to heary neutrino flavors in an energy regime

$$
m_{w} \ll \sqrt{s} \lesssim M_{1}
$$

where direct production, e.g. via the reaction

$$
\begin{aligned}
e^{-}+p \rightarrow & \mathcal{N}_{1}+X \\
& \stackrel{\downarrow}{\rightarrow} e^{+}+W-+X \\
& \rightarrow e^{+}+\mu^{-}+\bar{\nu}_{\mu}+X,
\end{aligned}
$$

is precluded.

The unique process, which satisfies these criteria is the two-electron (or two like-sign muon) reaction

$$
\ell \mp+\ell \mp \rightarrow W^{\mp}+W^{\mp}
$$

$$
\ell \mp=e^{\mp}, \mu \mp
$$

in the energy regime specified in eq. (4).

The double electron reaction has been studied extensively before, mostly in the framework of left-right symmetric models [3] , [4] , [5]

We wish to point out here the characteristic features, which can be traced back to a heavy neutrino flavor, that is exchanged in the crossed channel. First, there is the behaviour of the cross section [2] , [6]

$$
\begin{aligned}
& \sigma\left(\ell_{L}^{-} \ell_{L} \rightarrow W-W-; \mathcal{N}\right)= \\
& \sim \frac{1}{M(\mathrm{TeV})^{2}}\left(\frac{s}{M^{2}}\right)^{2}\left|\frac{h_{l}^{2}}{4 \pi}\right|^{2}\left(4.10^{5} \mathrm{fb}\right)
\end{aligned}
$$

In eq. ( 7 ) $h_{\ell} ; \ell=e, \mu, \tau$ denote three Yukawa couplings at the primary level $[6]$, where flavor mixing is determined by their directional cosines

$$
\begin{aligned}
& h_{\ell}=\xi_{\ell}|h|,\left.\left|h^{2}=\sum_{\ell}\right| h_{\ell}\right|^{2} \\
& \sum_{\ell}\left|\xi_{\ell}\right|^{2}=1
\end{aligned}
$$

The overall strength of the Yukawa couplings $|h|$ can well be of $O(.1-1)$. It determines together with the directional cosines $|\xi$,$| the absolute cross section$ for the reactions in eq. ( 6 )

The characteristic rise, displayed by the factor $\left(s / M^{2}\right)^{2}$, signals the onset of a hard process, governed by the lowest dimension of the effective Hamiltonian mediating the reaction at low energies, which is mass to the selent bower.

In addition to this characteristic energy dependence, the spectacular decay chanuels for the $W \mp W \mp$ system should provide crucial assistance for singling out process (6) : back-to-back decay's into the decay channels

$$
\begin{aligned}
& \rightarrow e^{-} \mu^{-}\left(\tau^{-}\right)+p_{\perp \text { miss }}, \\
& \rightarrow \mu^{-} \mu^{-}\left(\tau^{-}\right)+p_{\perp \text { miss }}, \\
& \rightarrow \tau^{-} \tau^{-}+p_{\perp \text { miss }} \\
& \rightarrow e^{-}\left(\mu^{-}, \tau^{-}\right)+\text {jet } \\
& \rightarrow \text { jet }+ \text { jet }
\end{aligned}
$$

where the jets reconstruct to $W$ masses. These signals cannot be missed by a good detector. Their signatures stand out from essentially all reactions of the Standard Model and its minimal extensions [7].

The masses of light neutrino flavors are systematically suppressed by the mixing with the heavy ones $\mathcal{N}_{1}, \mathcal{N}_{2}, \cdots$, with the mixing energy $O\left(m_{t}^{(\nu)}\right)$ much 
smaller than the energy difference briclged $[8],[9]$.

Their contributions to reaction ( 6 ) show no energy dependence in the kinematic regime considered (4), and are excluded by present limits on light neut rimo masses.

Neutrinoless double beta decay is the most sensitive domain for the effects due to Majorana masses of light neutrino flavors. It is also sensitive to contributions from heavy ones, but the distinction between light and heavy contributions can become apparent only through a systematic comparison of decay's involving various nuclei

$$
(A, Z) \rightarrow(A, Z \pm 2)+2 e^{\mp},
$$

which can be practically excluded

The light and heavy contributions reduce to the evaluation of nuclear transition matrix elenernts of the two operators

$$
\begin{aligned}
& \left\langle A, Z+2\left|\frac{m_{v} U_{z}^{2}}{1 \pi r_{u u}-d d}\right| A, Z\right), \quad \text { (light) } \\
& \left(A, Z+2\left|\frac{U_{N}^{2}}{M_{\mathcal{N}}} \delta^{3}\left(\vec{x}_{u u} \ldots d d\right)\right| A, Z\right) \text { (heavy) }
\end{aligned}
$$

In eq. $(10)$, the quantities $U_{v}, U_{N}$ symbolise complex mixing angles, and In eq. $(10)$, the quantities ${ }^{2}, N_{N}$ symbolise complex mixing angles, and the decay. $\vec{x}$ u $-d d$ denotes the relative position operator, which monitors the transition amplitude of two d-quarks in the mother nucleus $(A, Z)$ to two $u$ quarks in the daughter nucleus $(A, Z+2)$. In eq. $(10)$ we consider the case of double $e^{-}$decay for definitenes

Obviously, for one decay only, no distinction between light and heavy contributions is possible.

It has been pointed out $[10\}$ that similar restriction apply to all low.energy processes that are serisitive - in variable degrees - to these effects, such as

$$
\begin{array}{ll}
\mu & \rightarrow e+\gamma, 3 e \\
\mu^{-}+e^{+} & \leftrightarrow \mu^{+}+e^{-} \\
\mu^{-}+(A, Z) & \rightarrow e^{-}+(A, Z), e^{+}+(A, Z-2)
\end{array}
$$

Similasly, $\nu \bar{\nu}$ oscillations are sensitive to light Majorana masses only. Direct $\mathcal{N}_{M}$ production via $e^{-p}$ collisions limits experimental accessibility to masses of some $200 \mathrm{GeV} / c^{2}$ at HERA, to $800 \mathrm{GeV} / c^{2}$ at a presumptive LHC/LEP collider - neither of which is relevant for the present context. In an $e^{+} e^{-}$collider of the NLC generation, the reaction

$$
e^{+} \epsilon^{-} \rightarrow N_{M} \nu
$$

has no realistic discovery chance for a $\mathrm{TeV}$ mass.$N$, even if the necessary $\mathrm{CM}$ energy could be reached.

\section{Conclusions}

In an enveloping framework of unified interactions, operative upon breakdown of gauge symmetry at the unification scale $M$ GLT $\sim 10^{16} \mathrm{GeV}$, the generation of light neutrino masses - if any - and of lepton flavor violation finds its root in heavy neutrino flavors as illustrated in eq. (1).

Their lowest two masses, $M_{1}, M_{2}$, are constrained to lie above the band 1 $10 \mathrm{TeV}$. It is therefore logical to search for direct signatures of thiese heavy flavors, as distinct from indirect ones transmitted to the light ones, including not ably neutrino distinct from in
oscillations.

In the energy regime $m_{w} \ll \sqrt{s} \leq M_{1}$ (eq. 4), the lepton-flavor-violating reaction $e^{-} e^{-} \rightarrow W-W^{-}($eq 6 ) stands out through its unique signatures and clear interpretability. Recently developed ideas for high energy muon colliders, if proven feasible for like-sign incoming muon pairs, could enrich this capability through potentially selective access to a different mixing to heavy $\mathcal{N}$ flavors, with equally spectacular final states.

The crucial ratio $\sqrt{s} / M_{1} \leqslant 1$ and the lepton mixing parameters $\xi$ e will alone determine the success of these searches.

The next generation of lepton colliders, operated in a like-sign initial charge mode, has a unique chance to reveal first indications of heavy-flavored neutrinos prior to their discovery through direct production, which has to wait for the attainment of energies well beyond present accessibility. 


\section{References}

[1] P. Minkowski, Neutrino mass in the environment of 3 light and N' heary neutrino flavors, Bern University preprints BUTP-94/10 (1994) and BUTP-95/10

[2] C. A. Heusch and P. Minkowski, Nucl. Phys. B416 (1994) 3

[3] D. London, G. Bélanger and J.N. Ng, Phys. Leit. B188 (1987) 155

[4] T.G. Rizzo, Phys. Lett. B116 (1982) 23,

in Proc. of the Workshop on Physics and Experiments with linear $e^{+} e^{-}$Colliders, 26 - 30 April 1993, Waikoloa, Hawaii, F.A. Ilarris et al., eds., World Scientific, Singapore 1993, p. 520

[5] J. Maalampi, J. Pietilä and J. Vuori, Phys. Lett. B297 (1992) 327

[6] P. Minkowski, The decay chain E6 $\rightarrow$ SO10 $\rightarrow$ SU5 and lepton flavor violation, Bern University preprint BUTP-93/20 (1993),

in Proc. Worksbop on Physics and Experiments with Linear $e^{+} e^{-}$Colliders,

F. A. Harris et al., eds. , World Scientific, Singapore, 1993

[7] F. Cuypers et al., Phys. Lett. B325 (1994) 243

V. Barger el al., Phys. Rev. D50 (1994) 6704

[8] H. Fritzsch and P. Minkowski, Phys. Lett. B62 (1976) il

[9] M. Gell-Mann, P. Ramond and R. Slansky, in Supergravity, D. Freedman and

P. van Nieuwenhuizen edts., Nortb Holland, Amsterdam, 1979, p. 315

T. Yanagida, in Proc. of the Workshop on Unified Theory and Baryon Number

in the Universe, O. Sawada and A. Sugamoto edts., KEK, 'Tsukuba, 1979

R. Mohapatra and G. Senjanovic, Phys. Rev. Lett. 44 (1980) 912

[10] C. A. Heusch, SCIPP 94/24 (1994), to be published in Nucl. Phys. B 